\title{
Atypical Retinal Pigment Epithelial Hyperplasia and Glial Proliferation Masquerading as Progressive Recurrent Retinoblastoma: A Case Report Review and Clinicopathologic Correlation
}

\author{
Emily Zolfagharia, b Jonathan W. Kim ${ }^{a, b}$ Subramanian Krishnan ${ }^{b}$ \\ Patricia Chévez-Barrios ${ }^{c-g}$ Jesse L. Berry ${ }^{a, b}$ \\ ${ }^{a}$ The Vision Center at Children's Hospital Los Angeles, and ${ }^{b}$ USC Roski Eye Institute, Keck School of Medicine of the \\ University of Southern California, Los Angeles, CA, ' Department of Pathology and Genomic Medicine, Houston \\ Methodist Hospital, ${ }^{d}$ Retinoblastoma Center of Houston, ${ }^{e}$ Department of Ophthalmology, Baylor College of \\ Medicine, ${ }^{f}$ Department of Ophthalmology, Blanton Eye Institute, Houston Methodist Hospital, and ${ }^{9}$ Department of \\ Pathology and Laboratory Medicine, University of Texas, MD Anderson Cancer Center, Houston, TX, USA
}

\section{Established Facts}

- Active cancer recurrences arising from a scar are common in retinoblastoma, particularly in the 6 months after the end of chemotherapy treatment.

- Treatment of these recurrences requires consolidative therapy which can induce retinal pigment hyperplasia and glial proliferation.

\section{Novel Insights}

- A progressively advancing opaque white lesion in an eye undergoing treatment for retinoblastoma may be secondary to retinal pigment hyperplasia and glial proliferation.

- Optical coherence tomography technology can be useful to detect areas of true tumor activity in areas of recurrence.

\section{Keywords}

Retinal pigment hyperplasia - Glial proliferation •

Retinoblastoma $\cdot$ Recurrent retinoblastoma $\cdot$ OCT

\section{Abstract \\ Background: Recurrences of retinoblastoma tumors, partic- ularly scar recurrences, are a common phenomenon in the management of this cancer. Consolidative treatment with}

laser and cryotherapy are required for local control of disease. It is known that consolidative therapy can induce retinal pigment epithelium (RPE) hyperplasia and gliosis. Herein we report extensive RPE hyperplasia and gliosis during laser therapy for a focal scar recurrence, which presented as a progressive retinal opacification mimicking active retinoblastoma. Method: This is a retrospective case review. Results: A 2-month-old premature male was diagnosed with sporadic bilateral retinoblastoma (International Intraocular Retino-

\section{KARGER}

(c) 2017 S. Karger AG, Basel

E-Mail karger@karger.com

www.karger.com/oop
Jesse L. Berry, MD

Children's Hospital Los Angeles and the USC Roski Eye Institute

1450 San Pablo Street

Los Angeles, CA 90033 (USA)

E-Mail jesse.berry@med.usc.edu 
blastoma Classification [IIRC] group B in the right eye and IIRC group $A$ in the left eye). The patient underwent laser therapy for a focal recurrence which demonstrated a white lesion during therapy and was subsequently enucleated. While there was a focal recurrence and infiltration of the retina (seen both on optical coherence tomography and histopathologic section), the majority of the white, progressive lesion was from extensive RPE hyperplasia and gliosis secondary to laser therapy. Conclusion: Clinicopathologic correlation of the active recurrence and adjacent gliosis is demonstrated.

C 2017 S. Karger AG, Basel

\section{Introduction}

The emergence of new treatment modalities for retinoblastoma has improved globe salvage rates while also reducing systemic side effects; however, intraocular recurrences of this cancer are common, and, thus, it remains critically important for the ocular oncologist to monitor the eye closely for disease recurrence [1]. This is especially true in the first 6 months after intravenous or intra-arterial chemotherapy when recurrences from scars or new tumor formation in predisposed patients are more likely [2]. The treatment of these recurrences is often even more challenging than the initial management.

Retinal pigment epithelium (RPE) hyperplasia is a rare but known ocular side effect of therapy in retinoblastoma patients. It can occur as a result of RPE toxicity from intra-arterial and/or intravitreal chemotherapy, as well as from local laser therapy [3-6]. Indeed, laser photocoagu- lation, a common method used for the consolidative treatment of retinoblastoma, has been proven to induce RPE hyperplasia in and around the laser site on the retina using a mouse model $[7,8]$. However, most pigmentation patterns of RPE hyperplasia and/or hypertrophy are in contrast to retinoblastoma [3]; RPE changes often appear flat and darkly pigmented, occasionally presenting with a subretinal cleft, and do not tend to show significant or rapid growth, thus differentiating RPE hyperplasia from intraocular tumors [9]. Rarely, however, RPE hyperplasia may mimic an intraocular tumor $[3,10]$. Herein, we present an unusual case of atypical RPE hyperplasia occurring during aggressive laser treatment for a recalcitrant retinoblastoma scar recurrence. We detail the clinical and pathological features and the management utilized during treatment.

\section{Case Presentation}

A 2-month-old premature male born at 31 weeks was diagnosed with sporadic bilateral retinoblastoma (International Intraocular Retinoblastoma Classification [IIRC] group B in the right eye and IIRC group A in the left eye [11]). The patient was treated initially with 3 cycles of 3 -drug systemic chemotherapy per the previously published protocol at Children's Hospital Los Angeles [12]. Laser consolidation was initiated after the initial cycle, and the patient was followed at 4-weekly intervals.

Following therapy, the patient had stable disease until a recurrence was noted in the group B eye during examination under anesthesia at approximately 9 months of age (4 months after the last cycle of chemotherapy). Repeat laser consolidation was used as treatment for the recurrence with initial resolution of the tumor (Fig. 1a); however, at 22 months of age, the patient was observed to have an additional tumor recurrence from the same chorioreti-
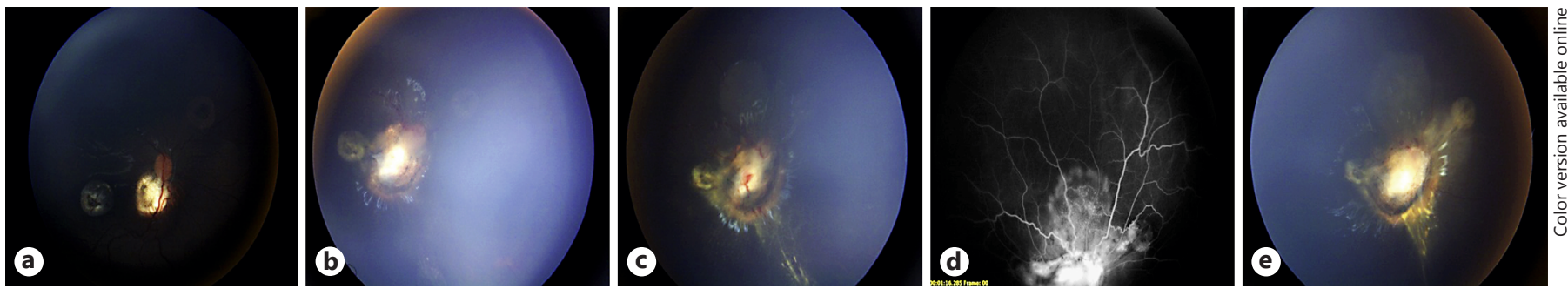

Fig. 1. Clinical Images. a Fundus photograph at 18 months of age. The patient's status is after 3 cycles of systemic chemotherapy. The right eye appears stable, and the retina is attached throughout the fundus. b Fundus photograph at 22 months of age at the time of the second recurrence; a new elevated mass adjacent to the nerve consistent with active retinoblastoma is seen. c Fundus photograph 2 months after $\mathbf{b}$ demonstrating progressive retinal opacification/thickening circumferential to the optic nerve disc. d Fluo- rescein angiography 1 month after $\mathbf{c}$. Photograph taken at $1 \mathrm{~min}$ $16 \mathrm{~s}$ demonstrating active leakage in the area of active recurring tumor directly over the optic nerve (active retinoblastoma) and very mild late staining in the area of retinal opacification without any distinct vascular changes. e Fundus photograph 1 month after d, taken prior to enucleation, which demonstrated a persistent active tumor nodule directly over the optic nerve with significant progression of the retinal opacification around the optic nerve.
RPE Hyperplasia Masquerading as a Recurrence of Retinoblastoma
Ocul Oncol Pathol 2018;4:116-121 DOI: $10.1159 / 000479741$ 

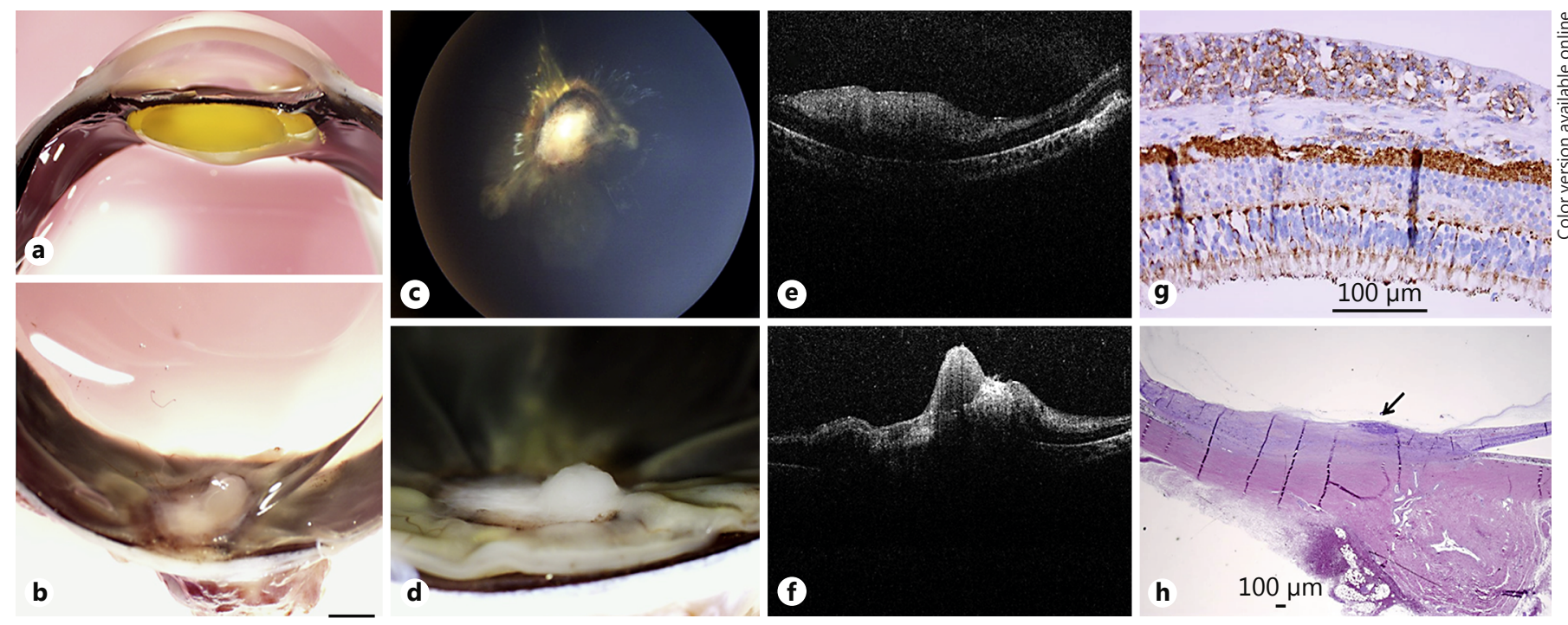

Fig. 2. Clinicopathologic correlation. a Composite of the macroscopic findings of the pupil-optic nerve section after taking the calottes. The anterior segment is unremarkable on top. b The vitreous is condensed and firmly attached to the white mass in the area of the optic nerve. c Clinical funduscopic photograph with a central white lesion associated with chorioretinal treatment scars. d Macroscopic photograph of the white scar and nodule to compare with histopathological findings (h). e Optical coherence tomography (OCT) with inner retinal thickening corresponding to focal active infiltrating retinoblastoma $(\mathbf{g})$. $\mathbf{f}$ OCT at the site of the scar/lesion showing a homogenous nodule of active disease on top

of an extensive chorioretinal scar. g Histopathologic section stained with immunohistochemistry using synaptophysin of the posterior retina adjacent to the scar. Notice that synaptophysin stains the synaptic regions of the retina and some of the photoreceptors but also the tumor in the inner retinal layers responsible for the thickening of the retina seen on OCT (original magnification $\times 10$ ). $\mathbf{h}$ Notice the darker basophilic nodule (arrow) corresponding to residual tumor, but the majority of the lesion represents RPE proliferation, glial proliferation, and fibrosis migrating from the sclera in this scar (HE stain; original magnification $\times 1.25)$.

nal scar, prompting continued laser consolidation every 3-4 weeks (Fig. 1b). Although the recurrence appeared to be responding to laser therapy, there was worsening subretinal fluid and eventually complete optic nerve obscuration secondary to the tumor and fibrotic scar. Moreover, the retina adjacent to the nodular recurrence exhibited increasing retinal whitening and opacification (Fig. 1c). Hand-held optical coherence tomography (OCT) demonstrated a nodular, homogenous mass overlying the optic nerve consistent with active retinoblastoma (Fig. 2f) along with associated inner retinal thickening and obliteration of the inner retinal layers (Fig. 2e). On funduscopic examination, an extensive area of the retina also appeared opaque and thickened. There were no vascular changes or hemorrhages, and fluorescein angiography was unrevealing with only mild staining in the area of whitening (Fig. 1d). After 8 weeks and 3 subsequent sessions of repeat laser consolidation, the nodular recurrence was responding, but the thickening and opacification of the retina had increased in size both clinically (Fig. 1e) and on OCT evaluation. Given the clinical findings, there was a concern for retinal infiltration of recurrent tumor. Thus, the right eye was enucleated after a prolonged treatment course including systemic chemotherapy and 17 sessions of laser consolidation for tumor recurrence.

Histopathological evaluation of the enucleated eye revealed macroscopic and microscopic correlation with funduscopic and imaging findings (Fig. 2). There was a poorly differentiated retinoblastoma with intraretinal growth and with an active focal and su-

perficial recurrence over the optic nerve associated with extensive regression and treatment effect (Fig. 2). The eye demonstrated extensive posttherapy effects with large chorioretinal scars with associated RPE proliferation and gliosis involving the optic nerve head and adjacent posterior retina (Fig. 2, 3). Immunohistochemistry showed that the active tumor was synaptophysin positive but negative for glial fibrillary acidic protein and both S100 protein and pancytokeratin. The bulk of the opaque white retinal mass was formed predominantly by RPE proliferation (pancytokeratin and S100 protein positive), fibrosis from the sclera, and glial proliferation (glial fibrillary acidic protein positive and S100 focally positive) (Fig. 4). Both the optic nerve margin and postlaminar optic nerve were negative for tumor. Thus, although clinically the patient appeared to have a large recurrent infiltrative retinoblastoma in the region not responding to local therapy, there was only a small nodular and infiltrative recurrence, and the majority of the tumor mass was actually formed by an atypical presentation of exuberant RPE hypertrophy and glial proliferation.

\section{Discussion}

Retinal pigment epithelial hyperplasia with gliosis can rarely manifest as a retinoblastoma tumor recurrence, particularly in patients receiving multiple rounds of laser 
Fig. 3. Histopathology. a Histopathological sections of the edge of the lesion with retina (black bar) partially replaced and thickened by residual/recurrent poorly differentiated retinoblastoma (white bar). Under the retina, there is the edge of the main mass formed by proliferation of retinal pigment epithelium (RPE) with pigmented and nonpigmented cells (HE stain; original magnification $\times 10$ ). $\mathbf{b}$ High magnification of the top of the nodule with residual/recurrent tumor in the scar composed by glial and fibrous tissue and deeper by the RPE proliferation (not seen in this picture) (HE stain; original magnification $\times 20$ ). c Near the top of the nodule adjacent to the recurrent tumor, there is condensation of vitreous $(V)$ firmly attached to the proliferation of glia, RPE, and fibrosis (HE stain; original magnification $\times 20$ ). d Glial proliferation with some RPE in atrophic retina adjacent to the scar (HE stain; original magnification $\times 20)$. e, $\mathbf{f}$ Edges of large RPE proliferation under the retina. Notice pigmented and nonpigmented cells (HE stain; original magnification $\times 20$ ).
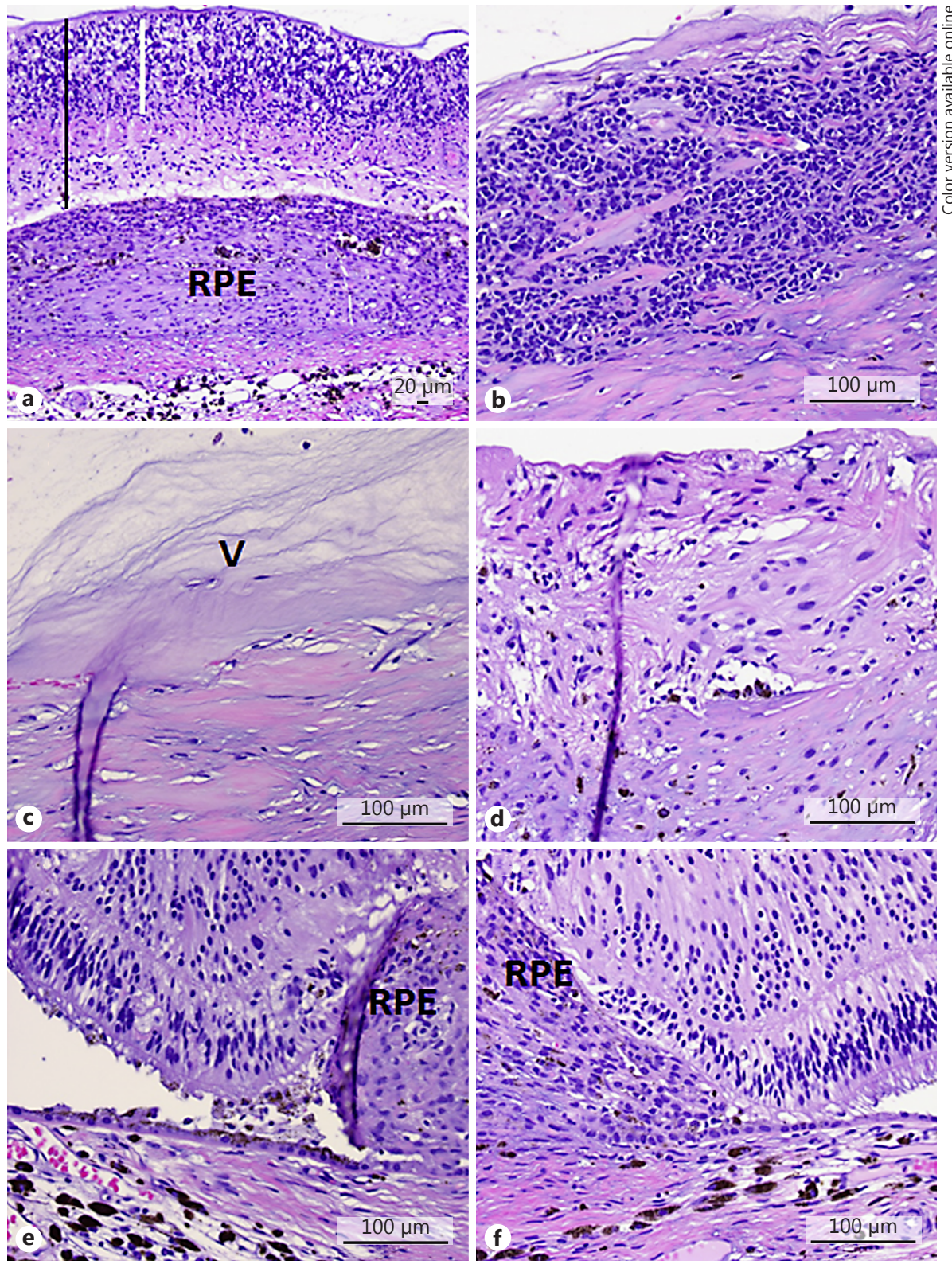

consolidation therapy. Although RPE hyperplasia, hypertrophy, and proliferation following laser therapy and chemotherapy in retinoblastoma patients have been documented, this case report demonstrates that, associated with glial proliferation, RPE hyperplasia can present as a progressive opaque white growth, thus mimicking the clinical presentation of active retinoblastoma, both on clinical exam and OCT imaging [3-6]. The knowledge of the phenomenon is especially important in patients with increasing retinal whitening after having received multiple rounds of laser consolidation therapy, which is known to induce RPE and glial proliferation. Another phenomenon that clinicians should consider in this setting is proliferative vitreoretinopathy, which has been reported to cause progressive whitening and vitreoretinal changes in retinoblastoma eyes, including tractional and rhegmatogenous detachment [13]. While rare, RPE hyperplasia can thus present a diagnostic dilemma for the treating ocular oncologist. Nonetheless, in the setting of retinoblastoma, the presence of any progressive thickening or whitening of the retina with documented increase in size must be considered active disease until proven otherwise.
RPE Hyperplasia Masquerading as a Recurrence of Retinoblastoma
Ocul Oncol Pathol 2018;4:116-121 DOI: $10.1159 / 000479741$ 

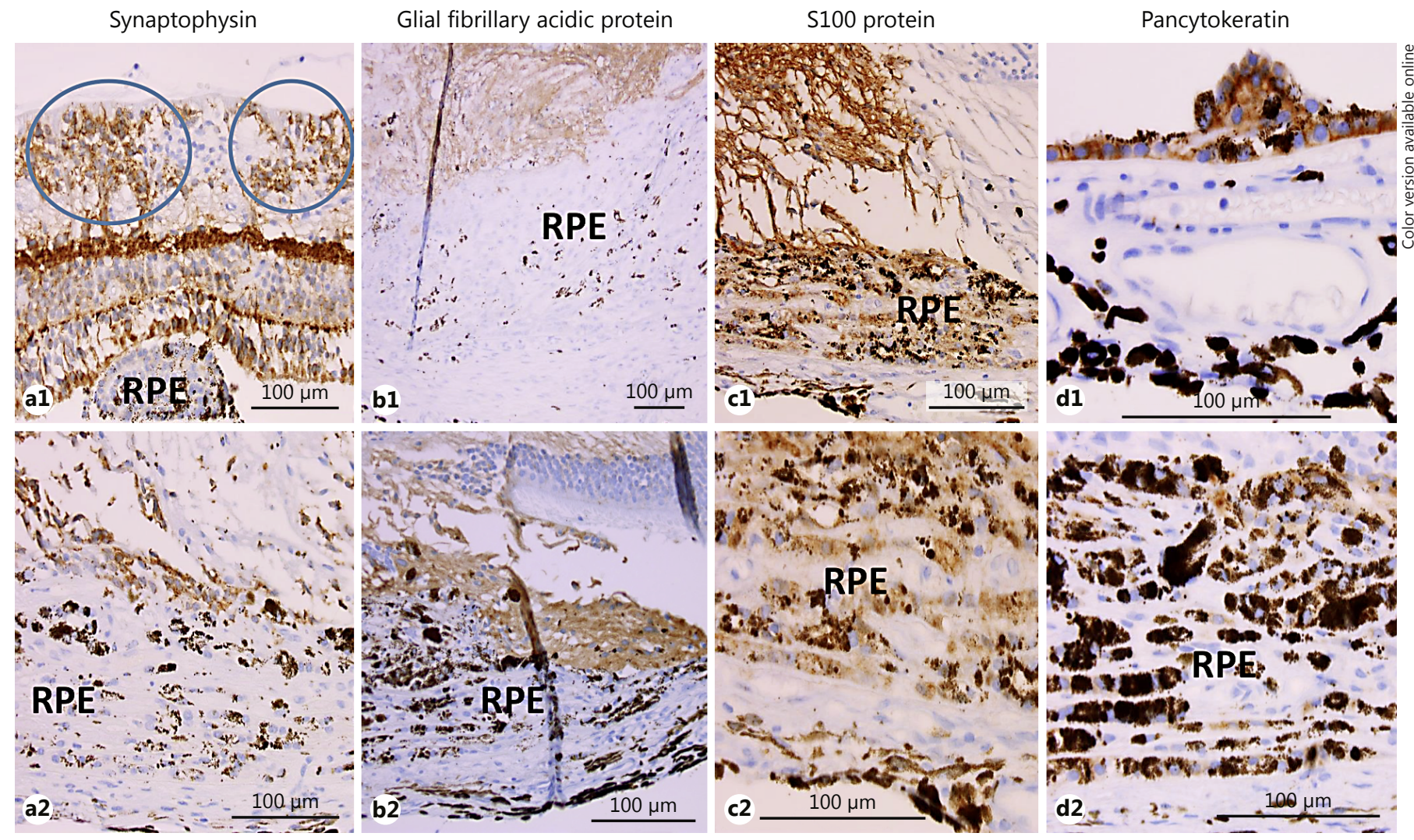

Fig. 4. Immunohistochemical staining of the scar and tumor. a Synaptophysin antibody. a1 Retina with staining of plexiform (synaptic) areas, some photoreceptors and tumor invading the inner retina (circle). The retinal pigment epithelium (RPE) proliferation under the retina is negative for this antibody (immunohistochemical stain with synaptophysin antibody and DAB chromogen; original magnification $\times 10$ ). a2 Edge of the scar shows that the majority of the lesion is RPE proliferation negative for synaptophysin with few entrapped photoreceptors and retina between the RPE and glial scar (top left) (immunohistochemical stain with synaptophysin antibody and $\mathrm{DAB}$ chromogen; original magnification $\times 20$ ). b Glial fibrillary acidic protein (GFAP) antibody. b1 Gliotic proliferation stains brown with GFAP and covers most of the RPE proliferation, which is negative for GFAP (immunohistochemical stain with GFAP antibody and DAB chromogen; original magnification $\times 10$ ). b2 Glial proliferation is seen at the edge of the RPE

proliferation under the retina (immunohistochemical stain with GFAP antibody and DAB chromogen; original magnification $\times 20$ ). c $S 100$ protein antibody. c1 Most of the RPE lightly stains with S100p, and glial proliferation is also positive. The remainder of the retina is negative (immunohistochemical stain with $\mathrm{S} 100 \mathrm{p}$ antibody and DAB chromogen; original magnification $\times 20$ ). c2 The main mass formed by the RPE proliferation stains with S100p (immunohistochemical stain with GFAP antibody and DAB chromogen; original magnification $\times 40$ ). d Pancytokeratin antibody. d1 A peripheral small RPE proliferation and adjacent RPE are strongly positive for cytoplasmic staining with pancytokeratin antibody. (immunohistochemical stain with pancytokeratin antibody and DAB chromogen; original magnification $\times 20$ ). $\mathbf{d} 2$ Most of the main mass of RPE proliferation is lightly positive with pancytokeratin (immunohistochemical stain with pancytokeratin antibody and $\mathrm{DAB}$ chromogen; original magnification $\times 20$ ).

\section{Statement of Ethics}

This study was approved by the Institutional Review Board at Children's Hospital Los Angeles.

\section{Disclosure Statement}

No conflicting relationship exists for any author.

\section{Funding Sources}

The Institute for Families, Inc., Children's Hospital Los Angeles, Los Angeles, CA, USA, Research to Prevent Blindness, New York, NY, USA, and the Larry and Celia Moh Foundation funded the study. 


\section{References}

1 Kim JW, Dunkel IJ, Abramson DH: Current management strategies for intraocular retinoblastoma. Drugs 2007;67:2173-2185.

2 Shields CL, Honavar SG, Shields JA, Demirci H, Meadows AT, Naduvilath T: Factors predictive of recurrence of retinal tumors, vitreous seeds, and subretinal seeds following chemoreduction for retinoblastoma. Arch Ophthalmol 2002;120:460-464.

3 Süsskind D, Hagemann U, Schrader M, Januschowski K, Schnichels S, Aisenbrey S: Toxic effects of melphalan, topotecan and carboplatin on retinal pigment epithelial cells. Acta Ophthalmol 2016;94:471-478.

4 Shields CL, Manjandavida FP, Arepalli S, Kaliki S, Lally SE, Shields JA: Intravitreal melphalan for persistent or recurrent retinoblastoma vitreous seeds: preliminary results. JAMA Ophthalmol 2014;132:319-325.
5 Muen WJ, Kingston JE, Robertson F, Brew S, Sagoo MS, Reddy MA: Efficacy and complications of super-selective intra-ophthalmic artery melphalan for the treatment of refractory retinoblastoma. Ophthalmology 2012;119: 611-616.

6 Shields CL, Bianciotto CG, Jabbour P, Griffin GC, Ramasubramanian A, Rosenwasser R, Shields JA: Intra-arterial chemotherapy for retinoblastoma: report No. 2, treatment complications. Arch Ophthalmol 2011;129:14071415.

7 Lee SH, Colosi P, Lee H, Ohn YH, Kim SW, Kwak HW, Park TK: Laser photocoagulation enhances adeno-associated viral vector transduction of mouse retina. Hum Gene Ther Methods 2014;25:83-91.

8 Lee SH, Kong YJ, Lyu J, Lee H, Park K, Park TK: Laser photocoagulation induces transduction of retinal pigment epithelial cells by intravitreally administered adeno-associated viral vectors. Hum Gene Ther Methods 2015; 26:159-161.
9 Shields CL, Manalac J, Das C, Saktanasate J, Shields JA: Review of spectral domain enhanced depth imaging optical coherence tomography of tumors of the retina and retinal pigment epithelium in children and adults. Indian J Ophthalmol 2015;63:128-132.

10 Maki JL, Marr BP, Abramson DH: Diagnosis of retinoblastoma: how good are referring physicians? Ophthalmic Genet 2009;30:199205.

11 Linn Murphree A: Intraocular retinoblastoma: the case for a new group classification. Ophthalmol Clin North Am 2005;18:41-53.

12 Zhu D, Berry JL, Ediriwickrema L, et al: Longterm outcomes of group B eyes in patients with retinoblastoma treated with shortcourse chemoreduction: experience from Children's Hospital Los Angeles/University of Southern California. Ocul Oncol Pathol 2015;2:105-111.

13 Hwang, Cindy S, et al: Proliferative vitreoretinopathy in treated retinoblastoma. Ophthalmol Retina 2017;1:165-172. 